\title{
Purification and characterization of recombinant murine endostatin in $E$. coli
}

\author{
Weon-Kyoo You', Seung-Ho So', Hyosil Lee', \\ Sun-Young Park', Mi-Ran Yoon², Soo-lk Chang ${ }^{2}$, \\ Hyun-Kyung Kim ${ }^{3}$, Young-Ae Joe $^{3}$, \\ Yong-Kil Hong ${ }^{3}$ and Soo-ll Chung ${ }^{1,4}$ \\ ${ }^{1}$ Mogam Biotechnology Research Institute, 341 Pojung-Ri, \\ Koosung-Myun, Yongin-City, Kyonggi-Do 449-910 Korea \\ ${ }^{2}$ Department of Biochemistry, College of Natural Sciences, \\ Chungbuk National University, Cheongju 361-763, Korea \\ ${ }^{3}$ Cancer Research Institute, The Catholic University of Korea, \\ Seoul, Korea \\ ${ }^{4}$ Corresponding author: Tel, 82-2-741-0611; \\ Fax, 82-331-262-6622; E-mail, rchung@greencross.com
}

Accepted 2 December 1999

Abbreviations: bFGF, basic fibroblast growth factor; CAM, cholioallantoic membrane; DMEM, Dulbeccos modified Eagles medium; BCS, bovine calf serum; FBS, fetal bovine serum; PBS, phosphate buffered saline; rmEndostatin, recombinant murine endostatin; LAL, Limulus Amebocyte Lysate; CD, circular dichroism

\begin{abstract}
Endostatin, a carboxyl-terminal fragment of collagen $\mathrm{XVIII}$ is known as an anti-angiogenic agent, that specifically inhibits the proliferation of endothelial cell and the growth of several primary tumor. We report here the purification and characterization of the recombinant murine endostatin (rmEndostatin) which was expressed in a prokaryotic expression system. This rmEndostatin has similar physiochemical properties of yeast-produced recombinant endostatin, and it also specifically inhibits the proliferation and migration of bovine capillary endothelial cells stimulated by basic fibroblast growth factor. The biological activity of rmEndostatin was also shown by its anti-angiogenic ability on the chorioallantoic membrane of chick embryo in vivo. In this article, we demonstrate the refolding and purification of rmEndostatin, expressed using E. coli system, to a biologically active and soluble form. In addition, these results confirm the activity of endostatin as a potent anti-angiogenic agent.
\end{abstract}

Keywords: recombinant endostatin, angiogenesis inhibition, prokaryotic expression system

\section{Introduction}

Angiogenesis, the formation of new capillaries from preexisting blood vessels, is involved in various pathological conditions such as primary tumor growth, metastasis, diabetic retinopathy and wound healing (Colville-Nash and Willoughby, 1997). Several angiogenesis inhibitors, developed as anti-cancer drugs, are in pre-clinical and clinical trials (Zetter, 1998). Recently, angiostatin and endostatin, produced by a primary Lewis lung carcinoma and hemangioma respectively, were reported to be potent angiogenesis inhibitors (O'Reilly et al., 1994; O'Reilly et al., 1997).

Endostatin, a $20 \mathrm{kD}$ carboxyl terminal fragment of collagen XVIII, is a specific inhibitor of the proliferation and migration of endothelial cells, and it also dramatically inhibits growth of the various primary tumors in mice (O'Reilly et al., 1997). Moreover, endostatin with repeat treatment on several tumors in mice was resulted in a persistent dormant state of the tumor (Boehm et al., 1997). The X-ray crystal structure of human and murine endostatin revealed that endostatin has a zinc binding site and a potent heparin binding site (Ding et al., 1998; Hohenester et al., 1998). In addition, this zinc binding of endostatin was reported to be essential for its antiangiogenic activity and molecular stability (Boehm et al., 1998; Sasaki et al., 1998). The anti-angiogenic mechanism of endostatin is less clear and the generation of endostatin from collagen XVIII in vivo has not been understood in detail. However, recent experiment showed that endostatin caused G1 arrest and induced apoptosis in various endothelial cells, and these effects were not seen in non-endothelial cell types (Dhanabal et al., 1999a; Dhanabal et al., 1999b).

There are several article of the cloning and expression of recombinant endostatin to active soluble form using yeast (Pichia Pastroris) expression system (Dhanabal et al., 1999a; Dhanabal et al., 1999c). Recombinant endostatin expressed in a prokaryotic system was also reported to have angiostatic activity but difficult to refold to a soluble form and tend to precipitate out of solution (O'Reilly et al., 1997; Dhanabal et al., 1999c).

In our study, we report the refolding and purification of recombinant murine endostatin (rmEndostatin), expressed in prokaryotic system, to a biologically active and soluble form. The purified rmEndostatin expressed in E. coli was comparable to that obtained using Yeast-expression system not only in physiochemical properties but also in anti-angiogenic activities shown by in vitro and in vivo angiogenesis assay systems. 


\section{Materials and Methods}

\section{Construction of the recombinant plasmid expressing endostatin}

The mc3b clone containing NC1 domain (Non collagenous C-terminal domain) of mouse $\alpha 1$ (XVIII) cDNA was a kind gift from Dr. B.R. Olsen. The sequence encoding endostatin was amplified by polymerase chain reaction (PCR) with Vent polymerase (New England Biolabs). The primer of 5'-end (ctaggatcccatatgCATACTCATCAGGACTTTCAG) contained $\mathrm{Nde}$ I site followed by annealing sequences of endostatin. The primer of 3'-end (atcggatccCTATTTGGAGAAAGAGGT) was designed to contain annealing sequence and a stop codon followed by Bam $\mathrm{HI}$ site. The PCR fragment was digested with $\mathrm{Nde}$ I and $\mathrm{Bam} \mathrm{HI}$ and ligated into pET-15b vector linearized with Nde I and $\mathrm{Bam} \mathrm{HI}$ and dephosphorylated by calf intestine alkaline phosphatase. The ligation mixture was first used to transform E. coli XL-1 Blue. After confirming the sequence, the recombinant plasmid was introduced into $E$. coli BL21 (DE3) for protein expression. The sequence of the constructs was confirmed by Chain termination DNA sequencing method using T7 sequenase version 2.0 DNA sequencing kit (Amersham).

\section{Expression and purification of murine endostatin in E. coli}

E. coli BL21 clones transformed with recombinant plasmid were grown in $3 \mathrm{~L}$ culture flask in LB media, and rmEndostatin was induced by $1 \mathrm{mM}$ IPTG. Cells were harvested by centrifugation for $30 \mathrm{~min}$ at $5,000 \mathrm{~g}$. Refolding and purification procedures of rmEndostatin were described briefly as follows; (i) cells were disrupted and the inclusion body (IB) was prepared by centrifugation and washed with deoxycholic acid. (ii) IB was then solubilized in Tris buffer ( $\mathrm{pH}$ 8.6) containing $7 \mathrm{M}$ guanidine- $\mathrm{HCl}$ and 50 $\mathrm{mM} \beta$-mercaptoethanol, and refolding was performed with rapid dilution in Tris buffer ( $\mathrm{pH}$ 8.6) containing $2.5 \mathrm{M}$ urea and redox-coupling agents such as reduced and oxidized glutathione. For purification, the refolded rmEndostatin fraction was applied to SP-Sepharose resin (pharmacia), which was pre-equilibrated with $20 \mathrm{mM}$ sodium phosphate buffer, $\mathrm{pH}$ 6.0. The resin was washed sufficiently with the equilibration buffer and then with the same buffer containing $0.5 \mathrm{M} \mathrm{NaCl}$ to remove nonspecific binding proteins. Refolded rmEndostatin fraction was eluted using the equilibration buffer containing $1 \mathrm{M}$ $\mathrm{NaCl}$. To remove His-tag portion of the rmEndostatin, thrombin cleavage was performed as previously described (Baubichon-Cortay et al., 1994). Thrombin was added to the purified protein (ratio of $1: 250$ ) in $20 \mathrm{mM}$ Tris buffer (pH 8.0) containing $150 \mathrm{mM} \mathrm{NaCl}$ and $2.5 \mathrm{mM} \mathrm{CaCl}_{2}$ for $30 \mathrm{~min}$ at $25^{\circ} \mathrm{C}$. The cleavage fragment was removed by using Superdex 75 HR 10/30 gel filtration resin (pharmacia).

\section{Characterization of recombinant murine endostatin (rmEndostatin)}

SDS-PAGE analysis was performed using precast 4 20\% polyacrylamide gels according to the manufacturers instructions (Novex). Samples analyzed under reducing conditions were dissolved in the SDS sample buffer containing $\beta$-mercaptoethanol. For western blot analysis, anti-rmEndostatin serum was obtained from rabbit immunized with purified rmEndostatin. Reverse phase (RP) HPLC analysis was performed on C18 column (260X $4.6 \mathrm{~mm}$, Vydac). A Gilson HPLC system was used with 5 95\% acetonitrile gradients for 45 min with a flow rate of $1 \mathrm{ml} / \mathrm{min}$. Circular dichroism (CD) spectra of rmEndostatin were measured on a JASCO J-715 spectropolarimeter using $1.0 \mathrm{~cm}$ path length cell at room temperature. Proteins were dissolved in $50 \mathrm{mM}$ sodium phosphate, $\mathrm{pH} 7.4$ at the final concentration of $0.1 \sim 0.15 \mathrm{mg} / \mathrm{ml}$, and filtered with $0.45 \mathrm{~mm}$ pore size filter unit before use. Protein concentration was measured using Lowry method.

\section{Cell culture}

Bovine capillary endothelial (BCE) cells were kindly provided by Dr. Lee H-T in Yonsei university (Lee et al., 1998). Cell culture was performed as previously described (Folkman et al., 1979). BCE cells were maintained in DMEM containing 10\% heat-inactivated BCS (bovine calf serum, Gibco$\mathrm{BRL}), 3 \mathrm{ng} / \mathrm{ml} \mathrm{bFGF}(\mathrm{UBI})$, and $1 \%$ antibiotics (GibcoBRL).

Lewis lung carcinoma cell and $\mathrm{NIH}$ 3T3 cell were purchased from American Type Culture Collection. Lewis lung carcinoma cells were maintained in DMEM containing $10 \%$ heat-inactivated FBS (fetal bovine serum, GibcoBRL) and $1 \%$ antibiotics (GibcoBRL), and NIH 3 T3 cells were maintained in the same media as that for BCE cells.

\section{Cell proliferation assay}

BCE proliferation assays were performed as previously described (O'Reilly et al., 1994). Briefly, cells were washed with PBS and were dispersed in a $0.05 \%$ of trypsin solution. A cell suspension was made with culture medium and the concentration was adjusted to $25,000 \mathrm{cells} / \mathrm{ml}$ after hemocytometer count. Cells were plated onto gelatinized 24 well culture plates $(0.5 \mathrm{ml} /$ well $)$ and were incubated $\left(37^{\circ} \mathrm{C}\right.$ in $\left.10 \% \mathrm{CO}_{2}\right)$ for $24 \mathrm{~h}$. After replacement of the media with $0.25 \mathrm{ml}$ of DMEM containing $5 \%$ BCS and $1 \%$ antibiotics, test samples were applied. After 30 min of incubation, media and bFGF were added to each well to obtain a final volume of $0.5 \mathrm{ml}$ of DMEM containing 5\% BCS, $1 \%$ antibiotics and $1 \mathrm{ng} / \mathrm{ml} \mathrm{bFGF}$. The plates were incubated for $72 \mathrm{~h}$, and then cells were dispersed in trypsin, resuspended in PBS and counted with hemocytometer. Each sample was tested in triplicate. Non-endothelial cell proliferation assays were also performed in the same condition as of the BCE cells.

\section{Cell migration assay}

Endothelial cell migration assay was accomplished with 
a modified Boyden chamber based assay ( $\mathrm{Ji}$ et al., 1998). Transwell polycarbonate membrane with 8- $\mu \mathrm{m}$ pore size (Costar) was coated with $0.1 \%$ gelatin (Gibco$\mathrm{BRL}$ ). After BCE cells were trypsinized and washed, cells were resuspended in DMEM containing $10 \%$ bovine calf serum and $10 \mathrm{ng} / \mathrm{ml} \mathrm{bFGF}$ at a concentration of $75,000 \mathrm{cells} / \mathrm{ml}$, and incubated at $37^{\circ} \mathrm{C}$ for $30 \mathrm{~min}$. During cell incubation, rmEndostatin with $5 \mathrm{ng} / \mathrm{ml}$ bFGF was loaded into the lower chamber with media. The gelatin-coated membrane filter was placed on top of the lower chamber and the top chamber was then attached. After cell incubation, BCE cells were loaded into the top chamber and incubated at $37^{\circ} \mathrm{C}$ for $16 \mathrm{~h}$. The chemotaxis chamber was then dismantled and the filter membrane was removed. The non-migrated cells were scraped from the upper surface of the membrane with cotton swabs three times. After rinsing with PBS, the membrane was fixed with $10 \%$ buffered formalin for $45 \mathrm{~min}$ and then stained with hematoxylin Gill No. 2 (Sigma) overnight. The number of migrated cells was counted on the hemocytometer and each sample was tested in quadruplicate.

\section{Chorioallantoic membrane (CAM) assay}

To determine anti-angiogenic activity in vivo, CAM assay was performed as previously described (Nguyen et al., 1994). Fertilized 3-day old eggs were incubated at $37^{\circ} \mathrm{C}$, $90 \%$ of humidity. After 2 day of incubation, $4 \sim 5 \mathrm{ml}$ of ovalbumin was extracted in the bottom of the eggs using syringe. After 3 day of incubation, fertilized eggs were made with window in the top of the eggs. After 4.5 day of incubation, thermonox (Nunc) coverslips (1/4 pieces) with test samples were applied on the CAM of individual embryos. After 7 day of incubation, embryos and CAMs were observed by means of stereomicroscope, and intralipose (white fat emulsion solution) was injected into the CAM using syringe for distinct discrimination of the blood vessel formation.

\section{Results}

\section{Expression and purification of rmEndostatin}

The recombinant murine endostatin (rmEndostatin) was expressed as inclusion body, and was refolded as a soluble form in the presence of chaotrophic agent (urea and guanidine- $\mathrm{HCl}$ ) and redox-coupling reagents such as glutathione (Figure 1A; lane 1 and 2). Refolded rmEndostatin was purified to a single peak using SP-sepharose and Superdex 75 column. The rmEndostatin was eluted at high salt fraction on SP-Sepharose column because endostatin is a very basic protein (calculated pl; 9.2). The rmEndostatin can be also purified using HeparinSepharose column due to its heparin-binding affinity (Dhanabal et al., 1999c). N-terminal His-tag region of rmEndostatin was removed by thrombin cleavage and gel filtration chromatography. The purified rmEndostatin
A

B

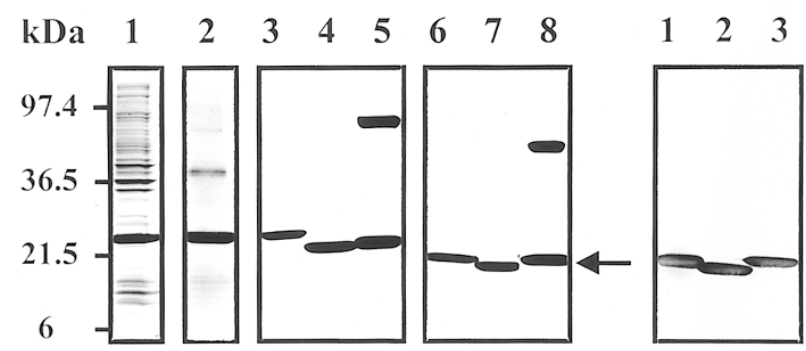

Figure 1. Expression and purification of the rmEndostatin in E. coli. A. SDS-PAGE analysis of purified rmEndostatin. Samples were loaded onto 4 20\% gradient gel followed by staining with Coomassie-Blue; lane 1: Cell lysate with $1 \mathrm{mM}$ IPTG, lane 2: Isolated inclusion body, lane 3 and 6: rmEndostatin purified with SP-Sepharose chromatography, lane 4 and 7 : rmEndostatin purified with Superdex 75 after thrombin cleavage, lane 5 and 8: Yeast-produced murine endostatin (Calbiochem). The upper band indicates albumin as stabilizer. Lane 6, 7 and 8 were performed under nonreducing conditions and other lanes were performed under reducing conditions, respectively. The arrow indicates purified rmEndostatin and Yeast-produced endostatin. B. Western blot analysis of purified rmEndostatin; lane 1: rmEndostatin purified with SP-Sepharose chromatography, lane 2: rmEndostatin purified with Superdex 75 after thrombin cleavage, lane 3: Yeast-produced murine endostatin. All lanes were performed under nonreducing conditions.

was confirmed with $\mathrm{N}$-terminal sequence analysis.

We obtained about $16 \mathrm{mg}$ of soluble purified rmEndostatin from $1 \mathrm{~L}$ of flask culture broth, which would be more than $10 \%$ of purification yield. Compared to the reported recombinant endostatin expressed in E. coli, where most of the endostatin was precipitated out of solution, we were able to successfully refold and purify the rmEndostatin expressed in prokaryotic expression system to a soluble forms with a high purification yield.

\section{Characterization of rmEndostatin}

The purified rmEndostatin has an apparent molecular weight of $20 \mathrm{kD}$ on the SDS-PAGE analysis under reducing conditions, and yeast-produced murine endostatin has also a similar molecular weight (Figure 1A). Both of the recombinant endostatin has a slight difference in mobility on the gel between reducing and non-reducing conditions as shown Figure $1 \mathrm{~A}$. The slight shift to a slower mobility after reduction is due to opening of the internal disulfide bonds. And these results were coincident with the previous report on the recombinant endostatin expressed in human embryonic kidney cells (Sasaki et al., 1998). In case of rmEndostatin where $\mathrm{N}$-term. His-tag region was removed, the band was shifted down on the gel.

Western blot analysis demonstrated that rmEndostatin and yeast-produced murine endostatin were also clearly recognized by an anti-rmEndostatin rabbit serum (Figure 1B). Structural aspect of rmEndostatin was confirmed by circular dichroism (CD) analysis. CD spectra of rmEndostatin were measured with a characteristic minimum at 

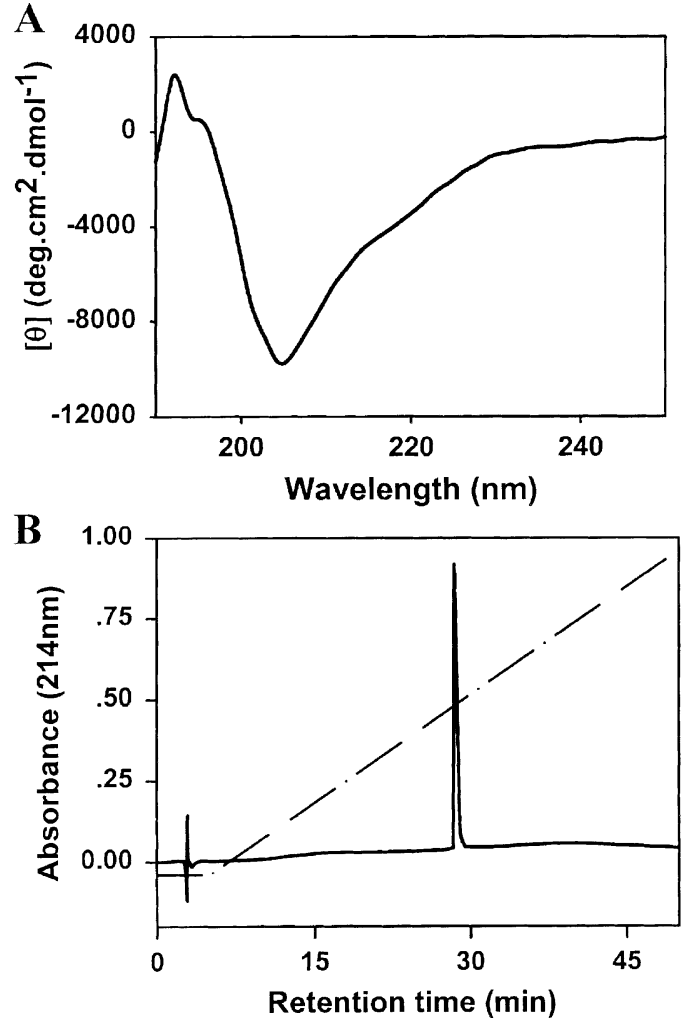

Figure 2. Characterization of purified rmEndostatin. $A$. CD spectra of rmEndostatin: $\mathrm{CD}$ spectra of rmEndostatin in $50 \mathrm{mM}$ sodium phosphate, $\mathrm{pH}$ 7.4, at room temperature. B. RP-HPLC analysis of rmEndostatin: purified rmEndostatin was injected on protein $\mathrm{C} 18$ reverse phase chromatography (Vydac) with a linear acetonitrile 5 95\% gradients for $45 \mathrm{~min}, 1 \mathrm{ml} / \mathrm{min}$ of flow rate. Solution $\mathrm{A}: 0.1 \%(\mathrm{v} / \mathrm{v})$ TFA (trifluoroacetic acid) in distilled water. Solution B: $0.085 \%(\mathrm{v} / \mathrm{v})$ TFA in acetonitrile. The dash and dotted line indicates the linear gradient of acetonitrile.

$205 \mathrm{~nm}$ as shown in Figure 2A. Secondary structure estimates with Chang's equation indicated the rmEndostatin to have about $10.9 \%$ of $\alpha$-helix and mostly $\beta$-sheet structure. These data were similar with the pervious report on recombinant endostatin (Sasaki et al., 1998). In the analysis of RP-HPLC, purified rmEndostatin showed a single peak as shown in Figure 2B, and there were no polymers or degraded fragments in the fraction of purified rmEndostatin. These results were confirmed with SDSPAGE analysis (Figure 1A)

All the above results indicate that the rmEndostain, which was expressed and purified in a prokaryotic expression system, has similar physiochemical properties of yeast-produced form.

\section{BCE cell assay}

To evaluate the function of rmEndostatin on endothelial cells, proliferation and migration assay of BCE cells were performed as previously described (O'Reilly et al., 1994; Ji et al., 1998). rmEndostatin inhibited bFGF-induced proliferation of BCE cells in vitro in a dose-dependent manner (Figure $3 \mathrm{~A}$ ). The $\mathrm{ED}_{50}$ of rmEndostatin in proliferation of BCE cells was approximately $0.2-0.3 \mu \mathrm{g} / \mathrm{ml}$ and it was comparable with that of the yeast-produced endostatin. There was no difference in anti-angiogenic activity whether $\mathrm{N}$-terminal His-tag region was intact or not (data not shown). In addition, there were no antiproliferative effects on non-endothelial cells such as tumor cell (Lewis lung carcinoma) and normal cell (NIH 3T3 cell) as shown in Figure 3B and $C$. And these results were in consistent with previous results (O'Reilly et al., 1997).

To exclude influence of endotoxin on BCE cells, the endotoxin of the purified rmEndostatin fraction was removed using ion-exchange column (DEAE-Sepharose and SP-Sepharose, Pharmacia). The endotoxin of rmEndostatin was determined to be less than $0.001 E U /$ well by LAL (Limulus Amebocyte Lysate) test, and there was no evidence of BCE cell detachment from the assay plates even at the highest concentration of rmEndostatin treated before cell counting.

rmEndostatin also inhibited migration of bFGF-induced
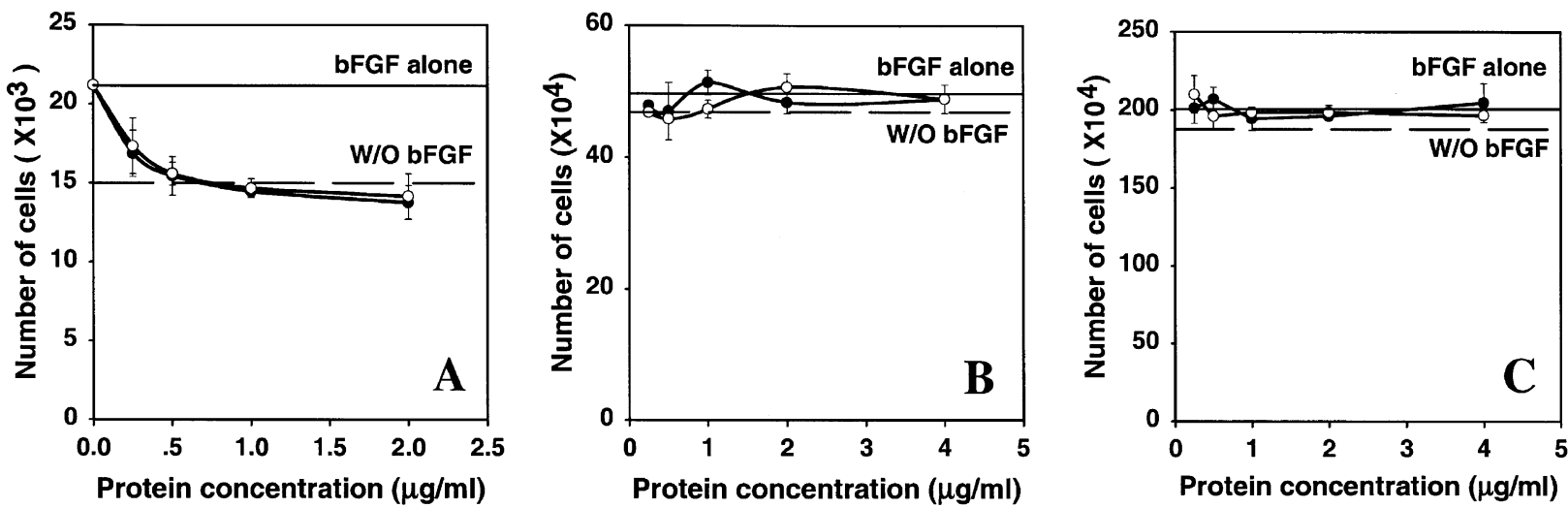

Figure 3. Cell proliferation assay in vitro. A. Purified rmEndostatin $(\bigcirc)$ and Yeast-produced murine endostatin $(\bullet)$ inhibit BCE cell proliferation in a dosedependent manner with a half-maximal dose, less than $0.3 \mu \mathrm{g} / \mathrm{ml}$. Solid line indicates the positive control with bFGF (1 ng/ml) alone, and dashed line indicates negative control. B and C. Purified rmEndostatin and Yeast-produced murine endostatin have no effect on the proliferation of non-endothelial cells such as Lewis lung carcinoma cell (B) and NIH 3 T3 cell (C), respectively 
A

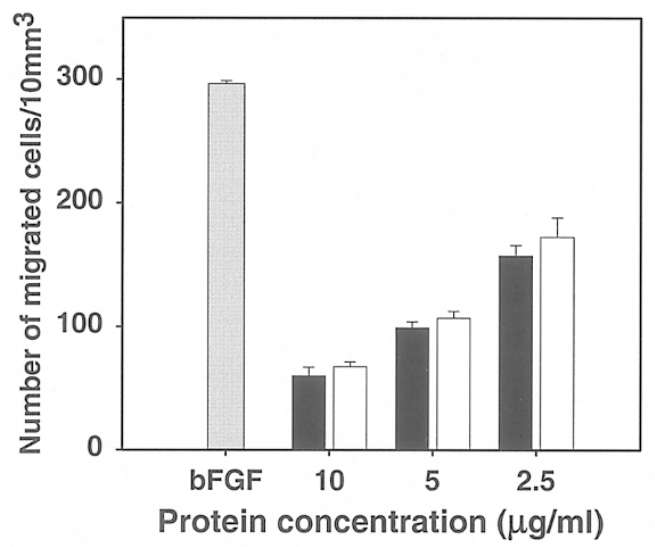

$\mathbb{B}$

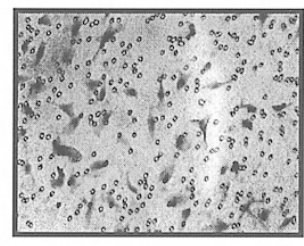

bFGF control
C

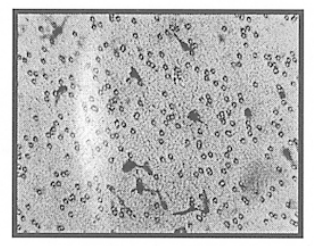

$\mathrm{bFGF}+\mathrm{rmEndostatin}$

Figure 4. Migration assay with purified rmEndostatin. A. Purified rmEndostatin $(\square)$ and yeast-produced murine endostatin ( $\square$ ) inhibit BCE cell migration on gelatin-coated transwell membrane $(8 \mu \mathrm{m})$ in a dose-dependent manner. B and C. Migrated BCE cells on the transwell membrane were stained with hematoxylin Gill No. 2 in the absence or presence of endostatin (10 $\mu \mathrm{g} / \mathrm{ml})$, respectively

BCE cells (Figure 4). The migration of BCE cells were inhibited by rmEndostatin in a dose-dependent manner (Figure 4A), and migration inhibition of BCE cells can be clearly seen through microscopy (Figure $4 B$ and $C$ ).

\section{CAM assay}

To determine whether rmEndostatin has an anti-angiogenic activity in vivo, anti-angiogenic activity of rmEndostatin was determined using chorioallantoic membrane (CAM) assay. rmEndostatin was able to potently inhibit the neovacularization in chick embryo in a dose-dependent manner with $\mathrm{ED}_{50}$ of $0.5 \mu \mathrm{g} / \mathrm{embryo}$ (Figure 5). When embryo was treated with more than $10 \mathrm{mg}$ of rmEndostatin, new blood vessel formation in chik embryo CAM was completely inhibited. Moreover, some chick embryo was not able to grow up to next developmental stage and died after all.

\section{Discussion}

Recently, anti-angiogenic agents are being watched as useful cancer therapy (Harris, 1998). Among these antiangiogenic agents, endostatin was recognized as one of the most powerful anti-cancer agents with fewer side effects. To develop endostatin for cancer therapy, it must

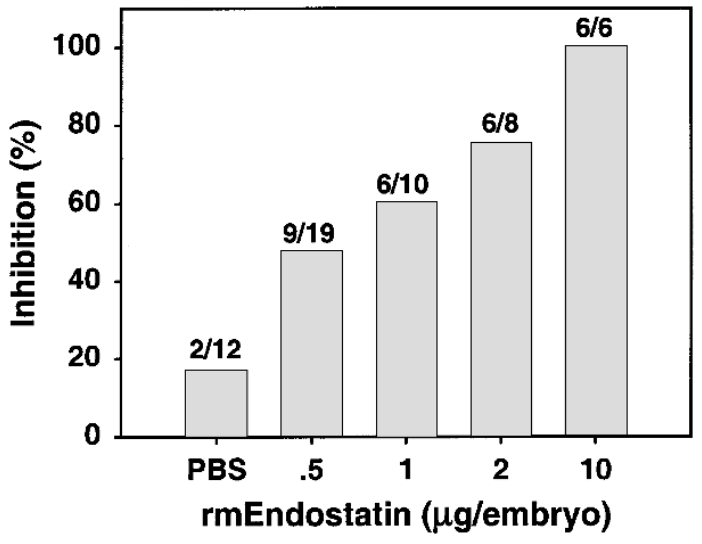

Figure 5. Anti-angiogenic effect of purified rmEndostatin on the chick CAM in vivo. The number of anti-angiogenic zone and the number of CAMs for each group are indicated above each bar. rmEndostatin inhibits angiogenesis on the CAMs in a dose-dependent manner. Inhibition (\%) of treated samples was measured with number of anti-angiogenic CAMs by total number of treated CAMs.

be produced in large quantity. Several expression systems of endostatin were reported (O'Reilly et al., 1997; Sasaki et al., 1998; Dhanabal et al., 1999c). One was eukaryotic system and the other was prokaryotic system. The recombinant endostatin, produced by eukaryotic expression systems (baculovirus and yeast), showed not only antiangiogenic activities in vitro and in vivo assay systems but also anti-cancer activties in various tumor grown animal models. However, there are some disadvantages of using the eukaryotic expression systems for large-scale production such as low production yield and high production cost. In this point, prokaryotic expression system is more useful and economical than eukaryotic system. There are some reports of endo-statin expressed in $E$. coli; however, the $E$. coli-derived recombinant endostatin was tend to precipitate and its final yield was reported to be less than $1 \%$. This low yield of recombinant endostatin expressed in $E$. coli was probably due to the refolding process of its protein. Generally, in the refolding process of recombinant protein, protein concentration and the $\mathrm{pH}$ of refolding buffer are very important factor to determine the yield of purification and the efficacy of refolding (Fischer et al., 1993).

In this study, we demonstrate the refolding and purification of the recombinant murine endostatin, expressed in prokaryotic system, to a soluble and biologically active form. Refolding of the solubilized inclusion body (IB) was accomplished by rapid dilution method in slightly basic refolding buffer containing $2.5 \mathrm{M}$ urea and redox-coupling agent with $1: 100$ of dilution factor. At this dilution factor, the protein concentration of this refolding fraction became less than $0.1 \mathrm{mg} / \mathrm{ml}$. Refolded and soluble rmEndostatin was purified with a single peak on SP-sepharose column at high salt elution step. SP-Sepharose column was useful than heparin-affinity column due to chemical stability to high concentration of urea, therefore we use SP-Sepharose 
instead of heparin-Sepharose to purify recombinant endostatin. The final yield of rmEndostatin was about $10 \%$ and it can be improved by adding high molecular weight protein stabilizer or basic amino acids such as polyethylene glycol or arginine, respectively (Rudolph and Lilie, 1996).

To determine if the $E$. coli-derived rmEndostatin was folded as the native endostatin, the purified rmEndostatin was characterized. The E. coli-derived rmEndostatin has a similar molecular weight and heparin binding ability as the yeast-produced endostatin. Moreover, poly-clonal antibody raised by rmEndostatin has immuno-chemical cross-reactivity with yeast-produced murine endostatin. Conformation analysis by $\mathrm{CD}$ also indicates rmEndostatin to have similar molecular structure of native or yeastproduced murine endostatin. In addition, the rmEndostatin has biological anti-angiogenic activities (determined by in vitro and in vivo angiogenesis assay) comparable to yeast-produced murine endostatin. These results show the possibility to produce recombinant active endostatin in large scale and high yield from $E$. coli. With this system, it would be easier to make quantity of endostatin to do further studies on mechanism as well as efficacy of the protein.

\section{Acknowledgement}

We are grateful to Drs. B. R. Olsen, S. K. Oh, and N. Fukai in Harvard Medical School for providing the collagen XVIII clone to us. We also thank Jong Hyouk Lee, Young Joe Kim, Byong Chol Ahn, Ki-Yong Kim, and Kwan-Yup Kang for their continued support. This research was supported by the national projects (98-G-08-03-A-26) of the Korean Ministry of Science.

\section{References}

Baubichon-Cortay, H., Baggeto, L. G., Dayan, G. and Pietro, A. D. (1994) Overexpression and purification of the carboxylterminal nucleotide-binding domain from mouse P-glycoprotein. J. Biol. Chem. 269, 22983-22989

Boehm, T., Folkman, J., Browder, T. and O'Reilly, M. S. (1997) Antiangiogenic therapy of experimental cancer does not induce acquired drug resistance. Nature 390, 404-407

Boehm, T., O'Reilly, M. S., Keough, K., Shiloach, J., Shapiro, R. and Folkman, J. (1998) Zinc-binding of endostatin is essential for its antiangiogenic activity. Biochem. Biophys. Res. Commun. 252, 190-194

Colville-Nash P. R. and Willoughby D. A. (1997) Growth factors in angiogenesis: current interest and therapeutic potential. Molecular Medicine Today 4, 14-23

Dhanabal, M., Volk, R., Ramchandran, R., Simons, M. and Sukhatme, V. P. (1999a) Cloning, expression, and in vitro activity of human endostatin. Biochem. Biophys. Res. Commun. 258,
345-352

Dhanabal, M., Ramchandran, R., Waterman, M. J., Lu, H., Knebelmann, B., Segal, M. and Sukhatme, V. P. (1999b) Endostatin induces endothelial cell apoptosis. J. Biol. Chem. 274, 11721-11726

Dhanabal, M., Ramchandran, R., Volk, R., Stillman, I. E., Lombardo, M., Iruela-Arispe, M. L., Simons, M. and Sukhatme, V. P. (1999c) Endostatin: yeast production, mutants, and antitumor effect in renal cell carcinoma. Cancer Res. 59, 189-197

Ding, Y. H., Javaherian, K., Lo, K. M., Chopra, R., Boehm, T., Lanciotti, J., Harris, B. A., Li, Y., Shapiro, R., Hohenester, E., Timpl, R., Folkman, J. and Wiley, D. C. (1998) Zinc-dependent dimers observed in crystals of human endostatin. Proc. Natl. Acad. Sci. U. S. A. 95, 10443-10448

Fischer, B., Sumner, I. and Goodenough, P. (1993) Isolation, renaturation, and formation of disulfide bonds of eukaryotic proteins expressed in Escherichia coli as inclusion bodies. Biotechnol. Bioeng. 41, 3-13

Folkman, J., Haudenschild, C. C. and Zetter, B. R. (1979) Long-term culture of capillary endothelial cells. Proc. Natl. Acad. Sci. U. S. A. 76, 5217-5221

Harris, A. L. (1998) Are angiostatin and endostatin cures for cancer? The Lancet 351, 1598-1599

Hohenester, E., Sasaki, T., Olsen, B. R. and Timpl, R. (1998) Crystal structure of the angiogenesis inhibitor endostatin at $1.5 \AA$ resolution. EMBO J. 17, 1656-1664

Ji, W. R., Castellino, F. J., Chang, Y., Deford, M. E., Gray, H., Villarreal, X., Kondri, M. E., Marti, D. N., Llinás, M., Schaller, J., Kramer, R. A. and Trail, P. A. (1998) Characterization of kringle domains of angiostatin as antagonists of endothelial cell migration, an important process in angiogenesis. FASEB J. 12, 1731-1738

Lee, T.-H., Rhim, T. and Kim, S. S. (1998) Prothrombin kringle2 domain has a growth inhibitory activity against basic fibroblast growth factor-stimulated capillary endothelial cells. J. Biol. Chem. 273, 28805-28812

Nguyen, M., Shing, Y. and Folkman, J. (1994) Quantitation of angiogenesis and antiangiogenesis in the chick embryo chorioallantoic membrane.Microvasc. Res. 47, 31-40

O'Reilly, M. S., Holmgren, L., Shing, Y., Chen, C., Rosenthal, R. A., Moses, M., Lane, W. S., Cao, Y., Sage, E. H. and Folkman, J. (1994) Angiostatin: a novel angiogenesis inhibitor that mediates the suppression of metastases by a Lweis lung carcinoma. Cell 79, 315-328

O'Reilly., M. S., Boehm, T., Shing, Y., Fukai, N., Vasios, G., Lane, W. S., Flynn, E., Birkhead, J., Olsen, B. R. and Folkman, J. (1997) Endostatin: an endogenous inhibitor of angiogenesis and tumor growth.Cell 88, 277-285

Rudolph, R. and Lilie, H. (1996) In vitro folding of inclusion body protiens. FASEB $J$ 10, 49-56

Sasaki, T., Fukai, N., Mann, K., Goring, W., Olsen, B. R. and Timpl, R. (1998) Structure, function and tissue forms of the Cterminal globular domain of collagen XVIII containing the angiogenesis inhibitor endostatin. EMBO J. 17, 4249-4256

Zetter, B. R. (1998) Angiogenesis and tumor metastasis. Annu. Rev. Med. 49, 407-424 